\title{
PARLARE IN PARLAMENTO. VITTORIO EMANUELE ORLANDO NELLA STORIA D'ITALIA
}

\author{
VALDO SPINI (*) \\ Nota presentata dal m.e. Silvio Beretta \\ (Adunanza del 16 aprile 2015)
}

SUNTO. - L'autore, rievoca un dialogo fra Vittorio Emanuele Orlando, liberale siciliano, e Piero Calamandrei, socialista liberale e democratico toscano, a proposito del linguaggio utilizzato nella vita parlamentare. Si sofferma quindi su di un saggio di Orlando il quale, traendo lo spunto da quel dialogo, tratta della natura del Parlamento democraticamente eletto, nel quale, tramite la delega degli elettori ai loro rappresentanti, si esprime la volontà popolare. La figura di Orlando viene quindi rievocata a partire dal suo ruolo durante la prima guerra mondiale fino alla sua attività di oppositore del fascismo.

$$
* * *
$$

ABSTRACT. - The author recalls a dialogue between Vittorio Emanuele Orlando, a Sicilian liberal, and Piero Calamandrei, a liberal and democratic Tuscan socialist, about the language used in parliamentary life. He then dwells on an essay by Orlando who, drawing inspiration from that dialogue, deals with the nature of the democratically elected Parliament, in which, through the delegation of voters to their representatives, the popular will is expressed. The figure of Orlando is then recalled starting from his role during the First World War up to his activity as an opponent of fascism.

Quando Tommaso Codignola, che dirigeva la casa editrice "Storia e Letteratura", mi chiese dei suggerimenti per pubblicare nella sua collana "Civitas" dei libretti agili, ma densi di significato, mi ritornò in

(*) Presidente dell'Associazione Istituzioni Culturali Italiane (AICI), Italia.

E-mail: valdospini@alice.it 
mente una lettura di molti anni prima. Lavoravo ad una rassegna dei contributi economici apparsi sulla rivista "Il Ponte", diretta da Piero Calamandrei ${ }^{1}$ ed avevo letto questo scritto, che era al di fuori della mia ricerca, ma da cui ero rimasto molto colpito. Ne caldeggiai quindi la ripubblicazione, che Tommaso Codignola (ed è suo merito) accettò molto volentieri. A mia volta aderii alla richiesta di scriverne la Prefazione cui rimando per una trattazione più completa.

"Parlare in Parlamento" riporta un dialogo tra due grandi spiriti: un vecchio liberale siciliano, Vittorio Emanuele Orlando, nato a Palermo nel 1860, poco dopo l'ingresso dei Mille e il più giovane, ma pur sempre maturo toscano, Piero Calamandrei, nato nel 1889, un socialista liberale e democratico, come quelli che poteva produrre la Firenze di Carlo Rosselli e del Partito d'Azione. Entrambi, va sottolineato, grandi giuristi nel loro specifico campo.

Il dialogo avviene nel 1951, poco tempo prima della morte di Orlando. Piero Calamandrei, già eletto alla Costituente per il Partito d'Azione, è stato rieletto alla Camera nel 1948 nella lista di Unità Socialista, frutto della convergenza tra i socialdemocratici di Saragat e gli ex azionisti fiorentini di Tristano Codignola, di cui lo stesso Calamandrei era un po' il nume tutelare (non c'era stata ancora la lacerazione tra gli azionisti fiorentini e Saragat che avverrà sulla "legge truffa" del 1953).

Calamandrei scrive ad Orlando: "Da quando io sono entrato in età più che matura nella vita parlamentare, ho sentito volare in due $\mathrm{o}$ tre anni nell'aula di Montecitorio più ingiurie e contumelie di quante in quarant'anni non ne abbia udite come avvocato nelle aule giudiziarie". E quindi il giurista fiorentino chiede al veterano Vittorio Emanuele Orlando di spiegargli allora perché si parli di "linguaggio parlamentare" o di "frase non parlamentare", insomma perché a tanta reverenza formale corrispondesse una prassi spesso volgare.

E Vittorio Emanuele Orlando si dichiara "sedotto" da questa richiesta e allarga il campo dell'argomento parlando delle origini del Parlamento e mettendo in evidenza come il segreto del successo della formula del Parlamento consista proprio nel parlare insieme, uomini

1 V. Spini, "Il discorso sull'economia e le scelte politiche (1945-56)", "Il Ponte” 1975, n. 11-12, pp. 1279-1373, ripubblicato con modifiche in V. Spini, "I socialisti e la politica di piano (1945-1964)", Firenze, Sansoni 1982, pp. 45-151. 
- oggi possiamo dire donne ed uomini - di tutti gli orientamenti ideologici e delle più varie provenienze sociali, e quindi dai linguaggi diversi e dissonanti ma costretti dal Parlamento a sedere insieme e a confrontarsi insieme. Insomma, da una dialettica anche esasperata arriva a formarsi una volontà unica, una decisione alla quale tutti devono conformarsi proprio perchè viene dal Parlamento democraticamente eletto. Per Orlando questo è il vero luogo in cui si esprime la volontà popolare, Certo, attraverso la delega, ma una delega fondata sulla rappresentanza. Una rappresentanza che per Orlando si doveva fondare sull'elezione diretta nel collegio elettorale, diffidente com'egli era, da buon liberale del prefascismo, nei confronti dei partiti e del loro filtro.

Il lettore gusterà la grande cultura che consente a Orlando di spaziare su varie esperienze parlamentari europee.

L'anziano statista arriva così a pronunciare un vero e proprio elogio del Parlamento e della sua insostituibile funzione democratica. E de resto memorabili erano stati anche i suoi discorsi di apertura e di chiusura dell'Assemblea Costituente.

Già questo basterebbe a indurre a leggere questo libretto, che ristampa lo scritto del politico e giurista siciliano, originariamente pubblicato sulla rivista di Piero Calamandrei "Il Ponte".

$\mathrm{Ma}$ è agevole riscontrare quanto questa tematica sia attuale, sia nelle motivazioni che portano Calamandrei a chiedere lo scritto ad Orlando (il turpiloquio che spesso sentiamo in Parlamento), sia nelle ragioni che portano Orlando a rispondergli riaffermandone la fondamentale funzione istituzionale nell'architettura della democrazia. Oggi siamo infatti in una vera e propria crisi della rappresentanza mentre il prestigio del Parlamento è caduto molto in basso. E questo non fa certamene bene né all'Italia né alla sua democrazia.

Ma l'attualità è anche un'altra. Possibile che il nostro paese abbia sostanzialmente cancellato dalla sua storia una figura politica così importante? È vero che Orlando commise l'errore fondamentale di abbandonare la Conferenza della Pace di Parigi con le conseguenze che ne seguirono. Ma è pure stato il Presidente della vittoria, o, come a me piace dire, della salvezza nazionale. È l'uomo che assume la Presidenza

2 V.E. Orlando, Il parlare in Parlamento, "Il Ponte", VII (giugno-luglio 1951), n. 6, pp. 567-585. 
del Consiglio dopo la rotta di Caporetto, che sostituisce nel comando supremo Luigi Cadorna con Armando Diaz, il quale a sua volta cambia la tattica militare e anche l'approccio politico passando dalla disciplina del terrore (le decimazioni e le fucilazioni) ad istituti come gli ufficiali addetti al morale della truppa. Se fosse passata la linea Cadorna la colpa di Caporetto sarebbe stata attribuita al disfattismo diffuso tra i soldati da parte di chi era contrario alla guerra, con le conseguenze repressive facilmente immaginabili. La linea di Orlando, sostituendo Cadorna e implicitamente riconoscendone gli errori militari, e d'altro lato confrontandosi con un Filippo Turati che, dopo la disfatta e di fronte al pericolo mortale che l'Italia correva, proclamava "la mia patria è sul Grappa”, impedì un'involuzione antidemocratica del nostro paese.

Assunta la carica di Presidente del Consiglio, Vittorio Emanuele Orlando interveniva alla Camera il 22 novembre 1917, commentando quanto era avvenuto e stava avvenendo sul Piave con queste parole: "La voce dei morti e la volontà dei vivi, il senso dell'onore e la ragione dell'utilità, concordemente, solennemente ci rivolgono adunque un ammonimento solo, ci additano una sola via di salvezza: resistere! resistere! resistere!".

Questo invito e questo ammonimento gli italiani forse lo conoscono di più per essere stato formulato proprio da Francesco Saverio Borrelli a Milano nel suo ultimo discorso da Procuratore Generale in occasione dell'apertura dell'anno giudiziario nel gennaio 2002, in tutt'altro contesto, in tutt'altre vicende. Ma il fatto che il procuratore Borrelli aggiungesse: "come su di una irrinunciabile linea del Piave" ci conferma che il diritto di autore è di Vittorio Emanuele Orlando.

Certo il fascismo aveva tutto l'interesse a cancellare la memoria di questo liberale che, da un iniziale voto favorevole al primo governo Mussolini, era passato a dare le dimissioni da deputato per protesta contro il famoso discorso del 3 gennaio 1925, quello in cui il Duce del fascismo si assume la piena responsabilità del delitto Matteotti. ${ }^{3} \mathrm{Fu}$ proprio Orlando, il successivo 16 gennaio, ad illustrare una mozione di censura al governo, presentata da lui stesso e da due altri ex presidenti del Consiglio dei Ministri, Giovanni Giolitti e Antonio Salandra. È faci-

3 Il deputato Giacomo Matteotti, segretario del Partito Socialista Unitario (Psu) era stato rapito ed ucciso il giorno 10 giugno del 1924 da una squadraccia fascista. 
le immaginare il clima di intolleranza con cui il suo intervento fu accolto da quella Camera. Non solo, ma in seguito a ciò gli squadristi gli devastarono il suo buen retiro, un villino al Saltino di Vallombrosa, nel comune di Reggello in provincia di Firenze.

Ancora, possiamo rilevare che, con grande dignità, Orlando rifiutò nel 1931 di prestare giuramento di fedeltà al fascismo e per questo dovette lasciare la sua cattedra universitaria di diritto costituzionale a Roma. Venne riammesso nel 1945, dopo la Liberazione e riprese l'insegnamento nel 1947-48. Dimostrò così una coerenza non consueta. E' quindi una figura che mantiene un asse di coerenza importante nella sua vita politica fino ad essere, nel 1948, il candidato di bandiera delle sinistre di Nenni e Togliatti per la Presidenza della Repubblica, in competizione con Luigi Einaudi che risulterà eletto con i voti della Dc e dei partiti di centro.

Ne viene, in conclusione, che dobbiamo ricucire il filo di un'identità nazionale democratica nei vari snodi della vita travagliata che l'Italia ha conosciuto dopo la sua unità. Grande è il ruolo della Presidenza della Repubblica in tale direzione. Dobbiamo rendere atto al ruolo che hanno svolto Carlo Azeglio Ciampi e Giorgio Napolitano nel corso delle celebrazioni del centocinquantesimo dell'unità nazionale. Ora sono di fronte a noi due anniversari: il settantesimo della Liberazione e il centenario della Prima Guerra Mondiale. Dobbiamo saperli condurre in questo spirito. 
